\title{
Bosons and Magnons in Ordered Magnets
}

\author{
U. KÖBLER* \\ Research Centre Jülich, Institute PGI, 52425 Jülich, Germany
}

(Received January 28, 2015)

\begin{abstract}
In earlier experimental studies we have shown that in accordance with the principles of renormalization group theory the spin dynamics of ordered magnets is controlled by a boson guiding field instead by exchange interactions between nearest magnetic neighbors. In particular, thermal decrease of the magnetic order parameter is given by the heat capacity of the boson field. The typical signature of boson dynamics is that the critical power functions either at $T=T_{\mathrm{c}}$ or at $T=0$ hold up to a considerable distance from critical temperature. The critical power functions of the atomistic models hold asymptotically at $T=T_{\mathrm{c}}$ or at $T=0$ only. In contrast to the atomistic magnons field bosons cannot directly be observed using inelastic neutron scattering. However, for some classes of magnets the field bosons seem to have magnetic moment and thus are able to interact directly with magnons. This interaction, although weak in principle, leads to surprisingly strong functional modifications in the magnon dispersions at small $q$-values. In particular, the magnon excitation gap seems to be due to the magnon-boson interaction. In this communication we want to show that for small $q$-values the continuous part of the magnon dispersions can be fitted over a finite $q$-range by a power function of wave vector. The power function can be identified with the dispersion of the field bosons. It appears that for low $q$-values magnon dispersions get attracted by the boson dispersion and assume the dispersion of the bosons. This allows for an experimental evaluation of the boson dispersions from the known magnon dispersions. Exponent values of 1, 1.25, 1.5, and 2 have been identified. The boson dispersion relations and the associated power functions of temperature for the heat capacity of the boson fields are now empirically known for all dimensions of the field and for magnets with integer and half-integer spin quantum number. These are two $2 \times 3$ exponent schemes.
\end{abstract}

DOI: 10.12693/APhysPolA.127.1694

PACS: 75.10.-b, 75.30.Ds, 11.10.-z, 11.10.Gh

\section{Introduction}

Since development of renormalization group (RG) theory, mainly by Wilson $[1,2]$, we know that the dynamics of ordered magnets is as for a continuous medium. Very surprisingly, spins and interactions between spins are not relevant for the dynamics (of the spins!). This is well known from the fact that the critical power functions of type $\left|T-T_{\mathrm{c}}\right|^{\varepsilon}$ either at $T=T_{\mathrm{c}}$ or at $T=0$ are identical for ferromagnets and antiferromagnets of the same symmetry class. Independence of the dynamics of the atomistic exchange interactions has the dramatic consequence that the actual value of the ordering temperature and the critical exponents as well are not determined by exchange interactions [3]. It was a major success of RG theory to have shown that a magnetic ordering transition occurs also when spins and interactions between spins are excluded from (spin) dynamics. In RG theory it is customary to call the boson driven magnetic ordering transition a stable fixed point (SFP) in order to distinguish it from the ordering temperature estimated by the atomistic models on the basis of the strength of the exchange interactions between nearest magnetic neighbors. The SFP always is at a somewhat lower temperature than the atomistic ordering transition. In other words, crossover of the dynamics from relevant atomistic interactions in the Curie-Weiss regime of the paramagnetic

\footnotetext{
*e-mail: u.koebler@fz-juelich.de
}

phase to the relevant boson field at lower temperatures shifts the transition temperature to a somewhat lower value.

Quite generally, the excitations of a continuous medium are delocalized bosons. In the ordered state these bosons are the relevant excitations to control the dynamics of the spins. Thermal energy is no longer with the interacting spins but has changed to the relevant boson guiding field. The spin system is, so to say, a thermodynamically passive system and follows the dynamics of the field. As a consequence, field theories instead of atomistic spin models are necessary for description of the dynamics of the boson guiding field [4].

RG theory could, however, not clarify the nature of the field quanta. Available field theories of the critical dynamics therefore rely on heuristic assumptions on the field properties [3, 4]. We have called all types of bosons of the magnetic continuum GSW bosons giving tribute to Goldstone et al. [5]. Experimental indication on the nature of the bosons is obtained from the observation that only in genuine Ising magnets the dynamics is atomistic $[6,7]$. This allows one to conclude that the field quanta are essentially magnetic dipole radiation emitted upon precession of the magnetic moments. In other words, the boson field has radiation character. Since Ising spins do not precess they cannot emit magnetic dipole radiation. The boson field gets not populated, and the dynamics is, in fact, atomistic. It was observed using elastic neutron scattering that the temperature dependence of the order parameters of the $2 \mathrm{D}$ Ising antiferromagnets $\mathrm{Rb}_{2} \mathrm{CoF}_{4}$ and $\mathrm{K}_{2} \mathrm{CoF}_{4}$ is in perfect 
agreement with Onsager's exact solution of the atomistic 2D Ising model [6-9]. Other atomistic spin models assuming three-dimensional spins seem not to be realized in nature.

Experimental distinction between atomistic dynamics and field dynamics is quite simple: the atomist critical power functions hold asymptotically at the critical point (either $T=0$ or $T=T_{\mathrm{c}}$ ) only while the observed boson determined critical power functions hold over a finite distance from critical temperature. Typical for Onsager's analytical solution of the $2 \mathrm{D}$ Ising model is that power series expansions can be made at $T=0$ and at $T=T_{\mathrm{c}}[8,9]$. Note that the famous critical exponent of $\beta=1 / 8$ for the order parameter of the $2 \mathrm{D}$ Ising model is the exponent of the leading term of a power series expansion at $T=T_{\mathrm{c}}$. Quite generally the critical range of the atomistic models is, so to say, zero.

In magnets with three-dimensional spin the critical power functions hold up to a considerable distance from SFP. $T=0$ also is a critical point in the sense that the dynamics near $T \approx 0$ is due to a boson field. This boson field is of main interest here. The critical power functions at $T=0$ are power functions of absolute temperature. This is well known from Debye's field theory of the low temperature heat capacity of solids. Typical for the field theory is that the $T^{3}$ function for the heat capacity of the field is independent of lattice structure and holds over a finite temperature range. In ordered magnets the validity range of the two universal power functions at $T=T_{\mathrm{c}}$ and at $T=0$ is very large. The two power functions overlap and give complete description of the magnetic order parameter for all temperatures [10-12].

The magnetic moment in the GSW boson field has much similarity with an electric dipole put into the electromagnetic radiation field. The electric dipole gets excited according to the spectral distribution of the electromagnetic radiation field. In the same way the non relevant spin system receives its thermodynamic behavior from the GSW boson field. Thermal decrease of the order parameter is according to the heat capacity of the boson field. Since emission and absorption of magnetic dipole radiation by precessing magnetic moments are weak processes, the mean free path of the field quanta is large. The approximation of freely propagating field quanta therefore is well justified. As a consequence, field theories of magnetism need to consider the energy degrees of freedom of the field exclusively. This is as for the Debye boson field that is independent on atomistic structures. The prominent thermodynamic observable of a field is its heat capacity. In magnetism spins are indicators of the heat capacity of the field. In other words, the heat capacity of the GSW boson field can be measured in two ways, either by the thermal decrease of the order parameter or by direct heat capacity measurements.

Because integer and half-integer spins precess differently, the emitted field quanta are different. The dynamics of the field therefore is different in magnets with integer and half-integer spin quantum number. In earlier publications we could establish the six universal exponents of the power functions of absolute temperature that describe thermal decrease of the magnetic order parameter. The six universality classes result from the three dimensionalities of the field and from whether the spin quantum number is integer or half-integer [10-12]. Note that the Debye boson field always is isotropic and the $T^{3}$ function for the heat capacity is observed also for anisotropic crystals. The GSW boson field has vector character and can assume any dimension.

Power functions of absolute temperature that hold over a finite temperature range, result when the dispersions of the responsible excitations are simple power functions of wave vector. This applies to freely (or ballistic) propagating field quanta only. A well known example is the electromagnetic radiation field. Since the dispersion of freely propagating photons is a linear function of wave vector, and the density of states is a quadratic function of frequency, the energy density of the field is given by the famous Stefan-Boltzmann $T^{4}$ function for all practical temperatures. One could call the Stefan-Boltzmann power function universal because it is due to a boson field and therefore holds over a large temperature range.

Direct experimental observation of the field bosons is difficult. Note that neutron scattering fails in detecting mass less bosons. Resonating magnetic waves in thin ferromagnetic films perhaps provide the only direct experimental technique to evaluate the dispersion of the GSW bosons [13]. Note that due to the macroscopic excitation process through irradiation with microwaves the standing magnet modes are resonating GSW boson states and not spin waves. For the excitation of standing magnetic modes it is decisive that the GSW bosons are essentially magnetic dipole radiation. The magnetic field vector of the rf-field acts on the rotating transverse spin components and thereby creates GSW bosons. Excitation of spin waves seems possible only by microscopic methods such as impact of neutrons. Moreover, only GSW bosons have a sufficiently large mean free path to travel freely over distances of a few hundred nm along film normal. So far standing magnetic wave experiments have been performed on thin ferromagnetic films with half-integer (effective) spin only. Note that ferromagnets with integer spin are extremely rare. In careful analyses of published standing wave experiments we could establish the three dispersion relations for all dimensions of the boson field $(d)$, though only for magnets with half-integer spin. The dispersion relations proved to be $\sim q, \sim q^{2}$ and $\sim q^{3 / 2}$ for $d=3, d=2$ and $d=1$, respectively [13]. Note that linear dispersion of the GSW bosons for $d=3$ is at variance with the quadratic dispersion of the magnons in isotropic ferromagnets. Moreover, in contrast to magnon dispersions boson dispersions always are perfectly gap-less.

Here we propose an alternative method to obtain information on the dispersions of the GSW bosons also for integer spin quantum numbers. For this method the interaction between GSW boson field and spin system 
(magnons) is decisive. Since the field bosons are essentially magnetic dipole radiation the coupling between field and spin system is given by the emission probability for magnetic dipole radiation and therefore should be weak. However, it turns out that the magnon dispersions are modified surprisingly strongly by interactions with the GSW boson field. A quantitative measure of this interaction is provided by the magnon excitation gap. This conclusion is suggested by the observation that no magnon gap occurs in isotropic magnets with half-integer spin. A prominent example for a vanishing magnon gap is metallic gadolinium $(S=7 / 2)$ [14]. No gap in isotropic magnets with half-integer spin fits the observed linear dispersion of the GSW bosons [13]. A linear dispersion means that the GSW bosons are mass-less and, as a consequence, cannot have magnetic moment. For direct interaction with magnons it seems necessary that the GSW bosons have magnetic moment (non-linear dispersion). Rather large magnon excitation gaps of the order of a few meV occur in isotropic magnets with integer spin. As we will see in those magnets the dispersion of the GSW bosons is $\sim q^{2}$. For quadratic dispersion GSW bosons are likely to have magnetic moment and thus can interact directly with magnons.

Not only the magnon gap is due to magnon-GSW boson interaction, magnon dispersions for small wave vector values also seem to be determined by the GSW bosons. In other words, we can expect that for small $q$ values the continuous part of the magnon dispersions agrees with the dispersion of the GSW bosons. If so, a simple power function of wave vector must hold over a finite $q$-range. As a proof of this idea we first discuss magnon dispersions of $\mathrm{MnF}_{2}[15,16]$. For a magnet as $\mathrm{MnF}_{2}$ with isotropic spin of $S=5 / 2$ and one-dimensional boson field we know from the independent method of standing magnetic wave experiments in metallic thin films that the dispersion of the field bosons is $\sim q^{1.5}$. This power function is confirmed by analyses of the magnon dispersions of $\mathrm{MnF}_{2}$ (Fig. 1).

Apparently, the dispersion relations of magnons and GSW bosons are attractive and assimilate. For small $q$-values magnons adapt to the dispersion of the GSW bosons. This opens the unique opportunity to evaluate the dispersion of the GSW bosons from the known magnon dispersions. We should note that attractive interactions can be observed also between the linear dispersion of the Debye bosons and the curved dispersion of the acoustic phonons. In many diamagnets, such as $\mathrm{NaI}$, the dispersion of the acoustic phonons with longitudinal polarization starts as a linear function of wave vector over a surprisingly large $q$-range [17]. The slope of the linear dispersion agrees with the measured sound velocities. For larger $q$-values a functional crossover to a different function of wave vector occurs. Surprisingly, the phonon dispersions adapt to the dispersion of the Debye bosons and not vice versa.

Quite analogous interaction effects can be observed in the magnon dispersions. A decisive difference to the

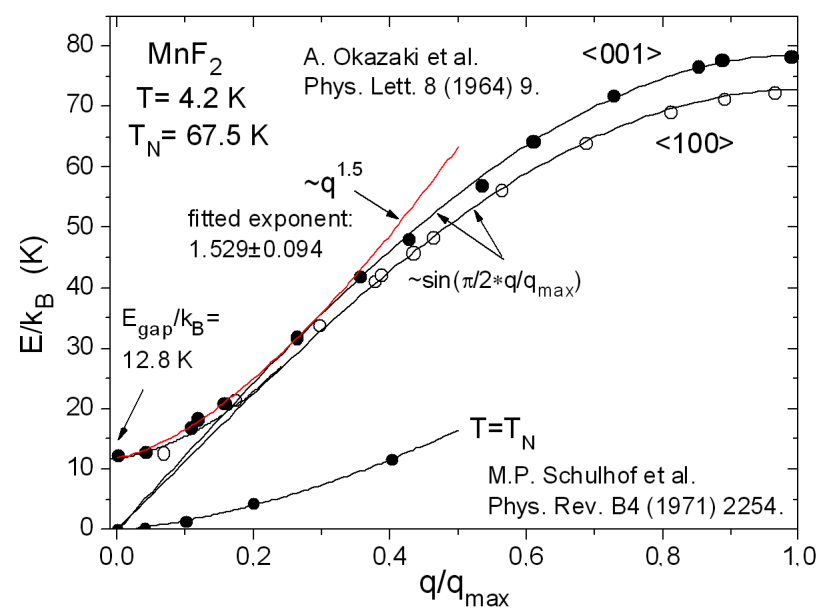

Fig. 1. Magnon dispersions of $\mathrm{MnF}_{2}$ transverse to tetragonal $c$-axis $(\langle 001\rangle)$ and parallel to $c$-axis $(\langle 001\rangle)$ [15]. For $q / q_{\max }>0.25$ both magnon branches follow sine function of wave vector. For $q / q_{\max }<0.25$ sudden deflection from sine function towards a magnon gap occurs. This analytical change indicates crossover from exchange defined to boson defined dispersion. For $q / q_{\max }<0.25$ magnon dispersion along symmetry axis $(\langle 001\rangle)$ is well described by $q^{1.5}$ function. At $T=T_{\mathrm{N}}$ magnon gap has closed but magnons with finite wave vector value persist into the paramagnetic phase [16].

phonon dispersions is that in magnetic systems there are different types of bosons with different dispersion relations. In particular, the dispersion of the GSW bosons depends on the dimensionality of the field. Different field dimensionalities result as a consequence of the axial radiation characteristic of the emission process of magnetic dipole radiation. In diamagnets the sources of the Debye bosons are unknown but they seem to have isotropic emission characteristic. As a consequence, it can be expected that the continuous part of the magnon dispersions starts by different power functions of wave vector. The experimental evaluation of the different power functions of wave vector will be the subject of this communication. The corresponding dimensionality of the field is inferred from the empirically known universal power function of absolute temperature in the thermal decrease of the order parameter (see Table I). For larger wave vector values a functional change occurs, and magnon dispersions are in better agreement with spin wave theory. As a conclusion, for low $q$-values magnon dispersions are boson defined but for large $q$-values they are exchange defined. It is evident that spin wave theory fails when the magnon dispersion agrees with the dispersion of the GSW boson field.

\section{Method of analysis}

$\mathrm{MnF}_{2}$ provides a suitable example to illustrate the different functionalities of boson field and atomistic exchange interactions. As can be seen in Fig. 1 magnon dispersions of $\mathrm{MnF}_{2}$ are very nearly isotropic [15, 16]. However, thermal decrease of the antiferromagnetic or- 
TABLE I

Universal power functions of absolute temperature describing thermal decrease of order parameter in dependence of the dimensionality of the field.

\begin{tabular}{c|c|c}
\hline \hline $\begin{array}{c}\text { Dimensionality } \\
\text { of field }\end{array}$ & $\begin{array}{c}\text { Integer } \\
\text { spin }\end{array}$ & $\begin{array}{c}\text { Half-integer } \\
\text { spin }\end{array}$ \\
\hline$d=3$ & $T^{9 / 2}$ & $T^{2}$ \\
$d=2$ & $T^{2}$ & $T^{3 / 2}$ \\
$d=1$ & $T^{3}$ & $T^{5 / 2}$
\end{tabular}

der parameter is according to $T^{5 / 2}$ function [18]. This is the universality class of magnets with $1 \mathrm{D}$ boson field and half-integer spin $(S=5 / 2)$ (see Table I). Since the $\mathrm{Mn}^{2+}$ ion has pure spin moment (the paramagnetic susceptibility is perfectly isotropic [19]) the extreme anisotropy of the boson field must come from the tetragonal rutile structure. Note that the anisotropic magnetic continuum has much similarity with the anisotropic elastic continuum. As a conclusion, in $\mathrm{MnF}_{2}$ magnon dispersions are isotropic but the GSW boson field is one-dimensional. Since magnons are not relevant for the dynamics it is evident that we have to classify $\mathrm{MnF}_{2}$ as a one-dimensional bulk antiferromagnet.

Due to the finite interaction between boson field and spin system (indicated by the magnon gap) the extreme anisotropy of the one-dimensional boson field gets transferred to the intrinsically isotropic spin system and aligns the spin moments rigidly along tetragonal $c$-axis as in a single domain. As a result, the spin-flop field of $\mathrm{MnF}_{2}$ is as large as $\approx 120 \mathrm{kOe}[20]$. This is an unusually large anisotropy field for a material with pure spin magnetism. As a consequence, only for a low dimension of the boson field the magnon gap is indicative of anisotropy. In cubic magnets with integer spin large magnon gaps occur but here the magnon gap does not mean anisotropy but only indicates strong interactions between GSW bosons and magnons (see discussion of $\mathrm{FeCl}_{2}$, Fig. 2) [21].

The most important detail in Fig. 1 is a functional change in the magnon dispersions. For wave vector values of $q / q_{\max }>0.25$ both magnon branches follow sine function of wave vector rather perfectly. This is in formal agreement with spin wave theory assuming nearest neighbor exchange interactions only. At about $q / q_{\max } \approx 0.25$ a sudden deflection from sine function towards a magnon gap at $q / q_{\max } \equiv 0$ can be noticed. It is evident that this analytical change cannot be explained by spin wave theory alone. Data points along tetragonal $c$-axis $(\langle 001\rangle$ direction) are well described by gap value plus $q^{1.5}$ power function. The fitted exponent is $1.529 \pm 0.094$. Using the independent method of standing magnetic waves in thin ferromagnetic films we have already identified the $q^{1.5}$ dispersion relation as due to the one-dimensional boson field in magnets with half-integer spin [13]. As a conclusion, along symmetry axis magnon energies of larger than gap energy agree with the dispersion of the GSW bosons up to $q / q_{\max } \approx 0.25$. We have every reason to assume that for $q / q_{\max }<0.25$ the magnon dispersion

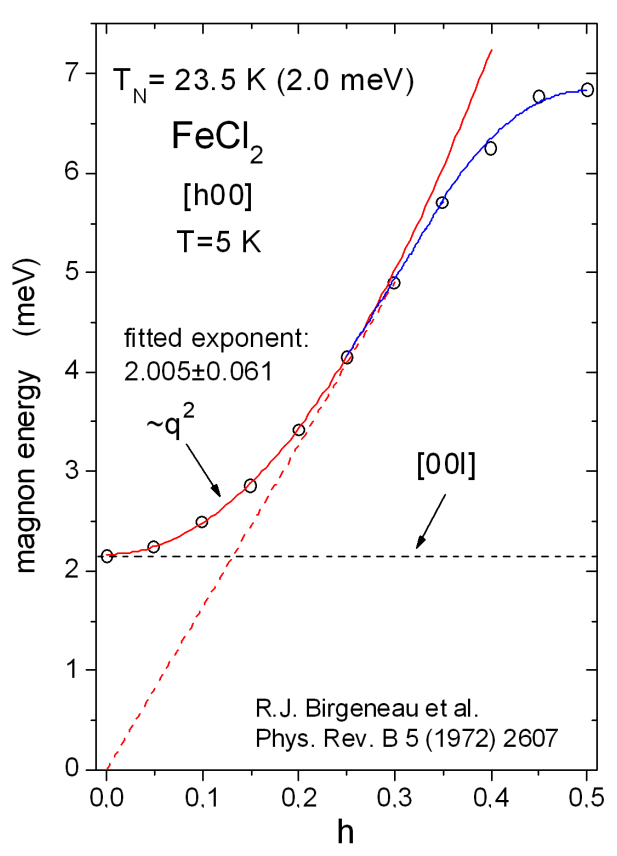

Fig. 2. Magnon dispersions of $\mathrm{FeCl}_{2}$ transverse to rhombohedral $c$-axis (open points) and parallel to $c$ axis (dashed horizontal line) [22]. Over a wide $q$-range magnon dispersion follows $\sim q^{2}$ function (fitted exponent: $2.005 \pm 0.061)$. The $q^{2}$ dependence is considered as dispersion relation of the isotropic GSW boson field in magnets with integer spin $(S=2)$. Due to magnon-GSW boson interaction (evidenced by the magnon gap) magnon dispersion assumes the dispersion of the GSW bosons up to a wave vector value of half of zone boundary.

gets attracted by the boson dispersion. In other words, for $q / q_{\max }<0.25$ magnon dispersions of $\mathrm{MnF}_{2}$ are boson defined but for $q / q_{\max }>0.25$ they are defined by atomistic exchange interactions. It is much surprising that the boson dispersions are much less perturbed by the interaction with magnons than vice versa. The dispersion of the boson field still exhibits simple power function of wave vector. We can assume that the stability of the boson dispersions is because the bosons are the thermodynamically relevant system. For a one-dimensional boson field power function fit should not be possible for the transverse magnon branch. This, however, cannot definitively be evidenced with the few available data points for $\mathrm{MnF}_{2}$. In the following we will use the boson defined part in the magnon dispersions for the evaluation of the missing boson dispersion relations for magnets with integer spin.

As a first example with integer spin we discuss antiferromagnetic $\mathrm{FeCl}_{2}$ with $S=2$ [22]. Magnon dispersions of $\mathrm{FeCl}_{2}$ are perfectly two-dimensional (see Fig. 2). There is no magnon dispersion observed along rhombohedral $c$-axis. Since the order parameter of $\mathrm{FeCl}_{2}$ decreases according to $T^{9 / 2}$ function, the boson field is $3 \mathrm{D}$ (see Table I) [21]. As a consequence, in spite of twodimensional magnon dispersions we have to classify $\mathrm{FeCl}_{2}$ as an isotropic antiferromagnet. Anisotropy of the boson 
field associated with the rhombohedral crystal structure evidently is small and not relevant. Isotropy of the relevant boson field is confirmed by the very small critical field of $\approx 11 \mathrm{kOe}[23]$. However, a characteristic measure of anisotropy is the spin flop field which is certainly much smaller than the critical field of $\approx 11 \mathrm{kOe}$.

The large magnon gap of $\mathrm{FeCl}_{2}$ with energy of $2.1 \mathrm{meV}$ $(\approx 24 \mathrm{~K})$ evidently does not fit the weak anisotropy field in $\mathrm{FeCl}_{2}$. According to the gap value the spinflop field should be of the order of $\approx 250 \mathrm{kOe}$. Because the boson field is isotropic (according to $T^{9 / 2}$ function of the order parameter) classical interpretation of the gap as anisotropy is incorrect. The gap only indicates that magnon-GSW boson interaction is strong. Nonrelevance of magnons is further evidenced by the fact that thermal decrease of order parameter (and magnetic heat capacity) does not follow exponential function of temperature but $T^{9 / 2}$ power function. Since the magnon gap is due to magnon-GSW boson interaction we expect that further increase of magnon energy should agree with the boson dispersion. In fact, it was early recognized [22] that up to a wave vector value of half of zone boundary magnon dispersion in the basal plane of $\mathrm{FeCl}_{2}$ is well described by $\sim q^{2}$ function (fitted exponent: $2.005 \pm 0.061$ ). The $\sim q^{2}$ dispersion we have to attribute to the isotropic GSW boson field in magnets with integer spin. Note that the excitations of a boson field always are gap-less.

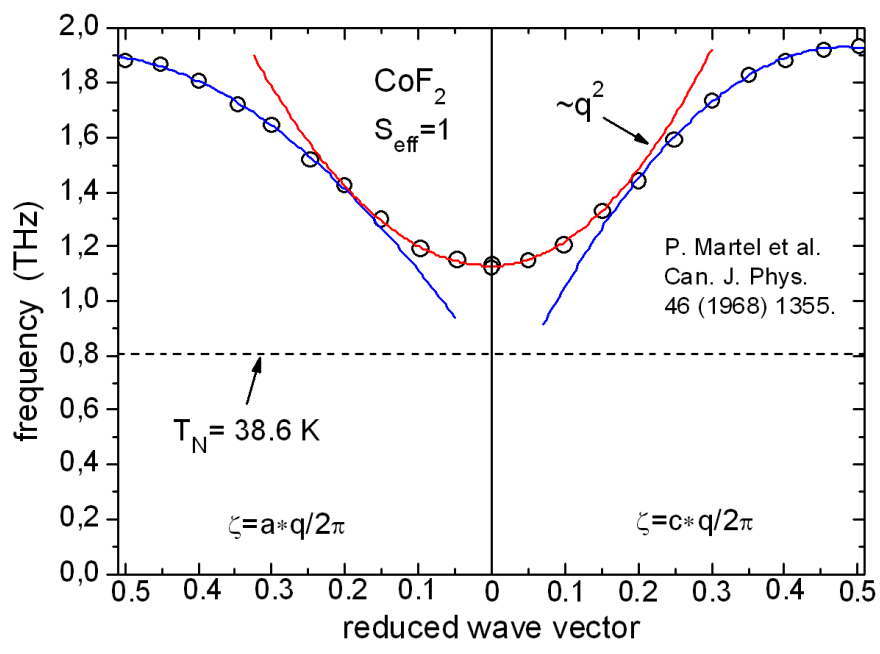

Fig. 3. Magnon dispersions of $\mathrm{CoF}_{2}$ transverse and longitudinal to tetragonal $c$-axis [24]. As for $\mathrm{MnF}_{2}$ (see Fig. 1) magnon dispersions are very nearly isotropic. The GSW boson field also is isotropic according to thermal decrease of order parameter by $\sim T^{9 / 2}$ function [21]. Due to a relevant crystal field interaction spin quantum number of the $\mathrm{Co}^{2+}$ ion is reduced from $S=3 / 2$ to effective spin of $S_{\text {eff }}=1[21,25]$.

$\mathrm{CoF}_{2}$ is another antiferromagnet to verify the $\sim q^{2}$ dispersion relation of the isotropic GSW boson field in magnets with integer spin [24]. Thermal decrease of the magnetic order parameter according to $\approx T^{9 / 2}$ function proves isotropic boson field and integer spin [21]. Inte- ger spin of the $\mathrm{Co}^{2+}$ is, however, not self-evident. Due to a relevant crystal field the spin of the $\mathrm{Co}^{2+}$ ion in $\mathrm{CoF}_{2}$ is reduced from $S=3 / 2$ to $S_{\text {eff }}=1$ [25]. Consistent with $S_{\text {eff }}=1$ is an observed saturation moment of $m=2.60 \pm 0.04 \mu_{\mathrm{B}} / \mathrm{Co}[26]$.

As for $\mathrm{FeCl}_{2}$ thermal equivalent of the magnon gap of $\mathrm{CoF}_{2}$ is larger than the ordering temperature (see Fig. 3). This shows that magnons have too high energies to be the relevant excitations. However, in contrast to rhombohedral $\mathrm{FeCl}_{2}$ magnon dispersions in tetragonal $\mathrm{CoF}_{2}$ are isotropic. Magnon dispersions transverse and longitudinal to $c$-axis follow $\sim q^{2}$ dependence up to $40 \%$ of the Brillouin zone boundary. Identical $q^{2}$ dependence further proves isotropic boson field.

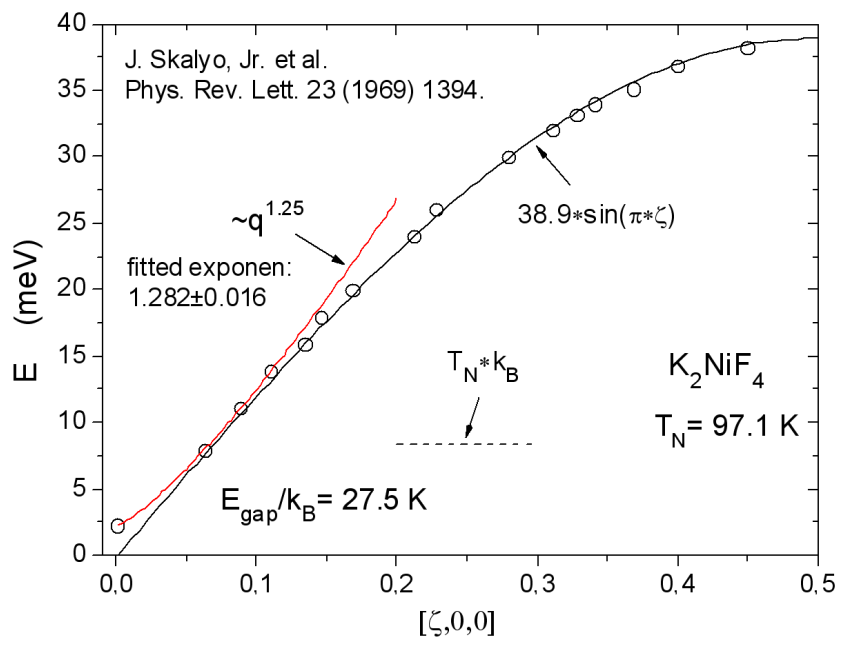

Fig. 4. Magnon dispersion in the basal plane of the tetragonal layered antiferromagnet $\mathrm{K}_{2} \mathrm{NiF}_{4}$ [27]. Over a large q-range the dispersion is well described by sine function of wave vector. For small $q$-values magnon energies above gap energy are well described by $q^{1.25}$ function (fitted exponent: $1.282 \pm 0.015$ ). This is the dispersion of the $2 \mathrm{D}$ boson field in magnets with integer spin.

A typical two-dimensional material is $\mathrm{K}_{2} \mathrm{NiF}_{4}$ with $S=1$ (see Fig. 4) [27]. In $\mathrm{K}_{2} \mathrm{NiF}_{4}$ magnon dispersions and GSW boson field are two-dimensional. According to the Mermin-Wagner theorem there should be no long range order in $\mathrm{K}_{2} \mathrm{NiF}_{4}$ [28]. The MerminWagner theorem supposes that the ordering transition is executed by exchange interactions. Since development of RG theory [1-4] we know that in magnets with threedimensional spin the phase transition is executed by the boson field. Under this condition there seems to be no restriction for the ordering transition. 2D dimensionality of the boson field is proven by $T^{2}$ function in the thermal decrease of the order parameter (see Table I) [21]. Figure 4 shows magnon dispersions in the basal plane of this tetragonal layered material [27]. It can be seen that over a considerable wave vector range magnon dispersion is well described by sine function. Note that thermal equivalent of magnon energy at zone boundary (the near neighbor interaction strength) is about $\approx 4.5$ times larger than the ordering temperature. In other words, the rele- 
vant boson field has shifted the ordering transition from zero (according to the Mermin-Wagner theorem) to a relatively high temperature.

Unfortunately, the magnon gap is relatively small compared to magnon energy at zone boundary. As a consequence of the weak magnon-GSW boson interaction the power function of wave vector in the continuous part of the spectrum holds over the limited wave vector range of $0<q<0.1$ only. The few useful data points in this $q$-range make evaluation of the exponent difficult. Nevertheless, $q^{1.25}$ dispersion relation can reasonably be identified. This dispersion relation we attribute to the $2 \mathrm{D}$ boson field in magnets with integer spin $(S=1)$.

Another example to verify the $q^{1.25}$ dispersion of the $2 \mathrm{D}$ boson field for integer spin is $\mathrm{Rb}_{2} \mathrm{MnCl}_{4}$ (see Fig. 5) [29]. Here again useful data for the evaluation of the exponent at low $q$-values are rather limited. The $q^{1.25}$ dispersion is, however, reasonably confirmed by the fitted exponent of 1.262 .

As for $\mathrm{CoF}_{2}$ crystal field interaction is relevant in $\mathrm{Rb}_{2} \mathrm{MnCl}_{4}$ and reduces the spin quantum number of the $\mathrm{Mn}^{2+}$ ion from $S=5 / 2$ to $S_{\text {eff }}=2$. Surprisingly, the spin of the $\mathrm{Mn}^{2+}$ ion with half-filled $3 d$ shell proves to be not very stable. A reduced spin of $S_{\text {eff }}=2$ also applies to $\mathrm{MnO}[21]$.

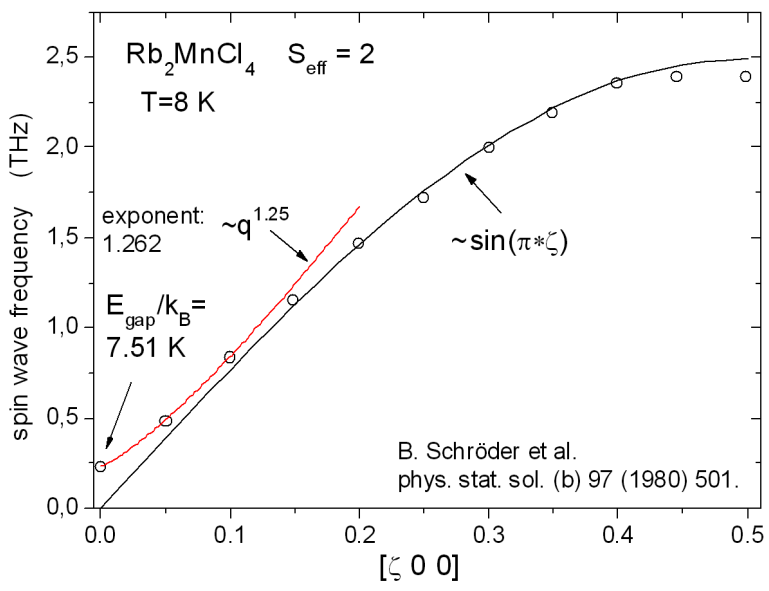

Fig. 5. Magnon dispersion in the basal plane of the 2D antiferromagnet $\mathrm{Rb}_{2} \mathrm{MnCl}_{4}$ [29]. The first three data points in the boson defined part of the spectrum allow evaluation of exponent 1.262. $q^{1.25}$ function is identified as dispersion relation of the $2 \mathrm{D}$ boson field for integer $\operatorname{spin}\left(S_{\text {eff }}=2\right)$.

Next we discuss $\mathrm{FeF}_{2}$ as a typical material with $1 \mathrm{D}$ boson field and integer spin $(S=2)$ [30]. 1D boson field is verified by $T^{3}$ function in the thermal decrease of the magnetic order parameter (see Table I). Condition for $1 \mathrm{D}$ boson field is an axial lattice structure. As $\mathrm{MnF}_{2}$ $\mathrm{FeF}_{2}$ with tetragonal rutile structure meets this condition. However, when anisotropy is weak and not relevant the boson field needs not be one-dimensional in axial crystals. $\mathrm{CoF}_{2}$ is an example for this. As for $\mathrm{FeCl}_{2}$ thermal equivalent of magnon gap is as large as Néel temperature in $\mathrm{FeF}_{2}$. Non-relevance of magnons again reveals from the fact that in spite of a large magnon gap thermal decrease of the order parameter is not according to exponential function of temperature but according to $T^{3}$ function. As can be seen in Fig. 6 the continuous part in the magnon excitation spectrum along $\langle 001\rangle$ direction is well described by $q^{1.5}$ function of wave vector up to $q / q_{\max } \approx 0.7$ (fitted exponent: $1.590 \pm 0.139$ ). Since the field is one-dimensional power function fit should be possible only for the magnon branch along tetragonal $c$ axis. This is difficult to verify explicitly. However it can be seen in Fig. 6 that magnon data transverse to $c$-axis $(\langle 100\rangle)$ can evidently not be described by a single power function of wave vector over the same $q$-range over which $q^{1.5}$ function holds along $\langle 001\rangle$ direction.

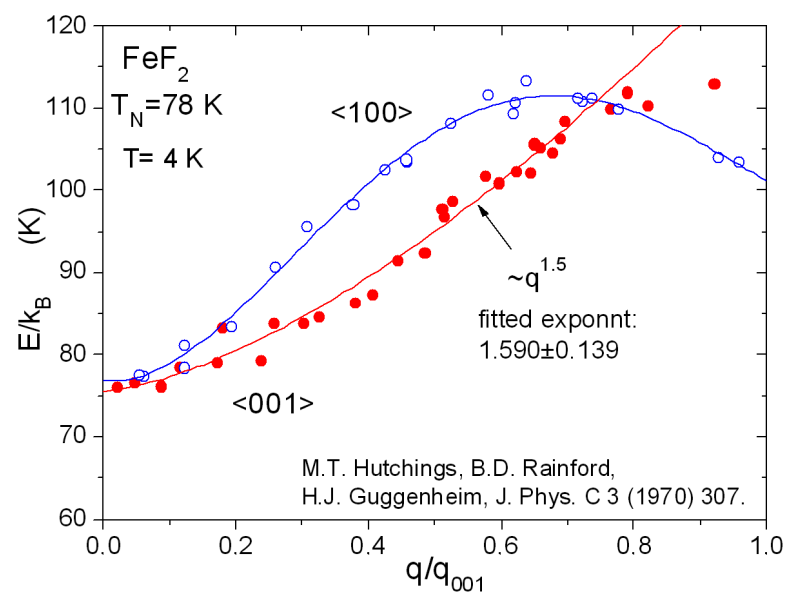

Fig. 6. Magnon dispersions of $\mathrm{FeF}_{2}$ along tetragonal $c$-axis $(\langle 001\rangle)$ and transverse to $c$-axis $(\langle 100\rangle)[30]$. $C$ axis data are well described by $q^{1.5}$ power function. This power function is identified as dispersion relation of the $1 \mathrm{D}$ boson field for integer spin $(S=2)$.

As a summary of the up to now obtained results it seems safe to conclude that for magnets with integer spin the dispersions of the GSW bosons are $\sim q^{2}, \sim q^{1.25}$ and $q^{1.5}$ for field dimensions of $d=3, d=2$ and $d=1$, respectively (see Table II). We can assume that these dispersion relations of the field will continue up to much larger energies than can be sampled in the magnon dispersions.

TABLE II

Dispersion relations of the GSW bosons in dependence of the dimensionality of the field.

\begin{tabular}{c|c|c}
\hline \hline $\begin{array}{c}\text { Dimensionality } \\
\text { of field }\end{array}$ & $\begin{array}{c}\text { Integer } \\
\text { spin }\end{array}$ & $\begin{array}{c}\text { Half-integer } \\
\text { spin }\end{array}$ \\
\hline$d=3$ & $q^{2}$ & $q$ \\
$d=2$ & $q^{1.25}$ & $q^{2}$ \\
$d=1$ & $q^{1.5}$ & $q^{1.5}$
\end{tabular}

The three dispersion relations of $\sim q^{2}, \sim q^{1.25}$ and $\sim q^{1.5}$ in magnets with integer spin are retrieved in many other materials but the exponents can occur in an unexpected context. One problem is the effective spin which can be lower than the free ion value. Furthermore, identification of the associated dimension of the field can be ambiguous. 
Let us note that the dimension of the field can depend sensitively on strain in the sample $[18,31]$. Small axial lattice distortions of nominally cubic magnets can lower the dimensionality of the field from $d=3$ to $d=1$. Moreover, for distorted (anisotropic) cubic magnets the dimensionality class is identical with the dimensionality class of homogeneous 2D magnets. An example of this seems to be USb [32, 33]. Nominally, the lattice of USb is cubic. According to an observed saturation magnetic moment of $\approx 2.8 \mu_{\mathrm{B}} /$ uranium atom the spin is $S=1$ [32]. As can be seen in Fig. 7 magnon data of Hälg and Furrer [32] and of Lander and Stirling [33] follow $\sim q^{1.25}$ function (fitted exponent over both data sets: $1.203 \pm 0.056$ ). As a conclusion, USb cannot be perfectly cubic.

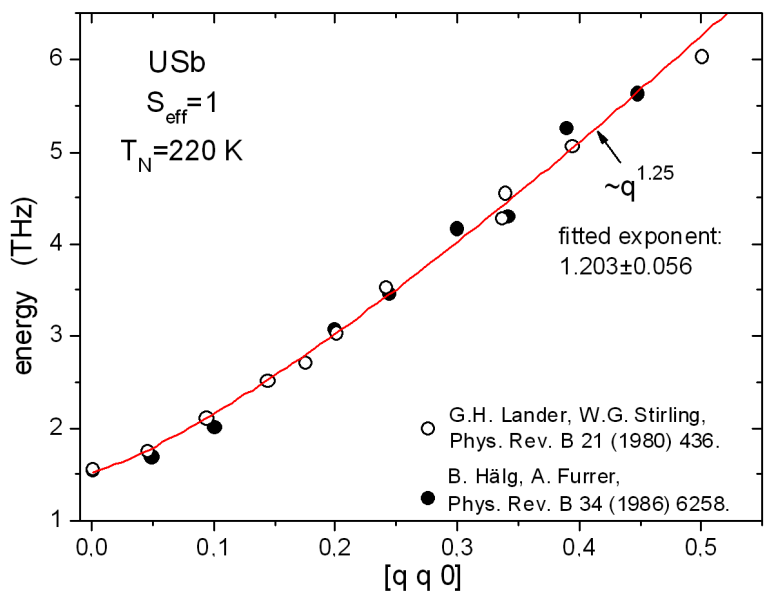

Fig. 7. Magnon dispersion along face diagonal of nominally cubic USb $[32,33]$. Fit over both data sets results in exponent $1.203 \pm 0.056$. This value is consistent with 1.25 . Since the exponent of 1.25 applies to magnets with homogeneous $2 \mathrm{D}$ boson field and to magnets with anisotropic 3D boson field as well, the lattice structure of USb cannot be perfectly cubic but must be distorted.

Another example of a relevant lattice distortion is $\mathrm{K}_{2} \mathrm{MnF}_{4}$ (Fig. 8) [34]. Nominally the materials of the $\mathrm{K}_{2} \mathrm{NiF}_{4}$ structural family are $2 \mathrm{D}$. We should note that lattice distortions much depend on sample preparation and, as a consequence, can be characteristic for the individual specimen only. Deviations from ideal tetragonal lattice symmetry have been evidenced for $\mathrm{Rb}_{2} \mathrm{FeF}_{4}$ using the Mössbauer spectroscopy [35]. Instead by $T^{2}$ function expected for unperturbed 2D symmetry the order parameter of the $\mathrm{Rb}_{2} \mathrm{FeF}_{4}$ sample investigated in [35] decreases by $T^{3}$ function [21]. This is the universality class of distorted 2D magnets and of homogeneous $1 \mathrm{D}$ magnets with integer spin $(S=2)$. A similar distortion seems to apply to the $\mathrm{K}_{2} \mathrm{MnF}_{4}$ sample investigated in [34] (Fig. 8). Thermal decrease of the order parameter is by $T^{5 / 2}$ function proving 1D boson field and half-integer spin $(S=5 / 2)$. Fit of a power function through the first three data points of the magnon dispersion results into exponent of 1.62 . This exponent can be considered as consistent with 1.5. As a consequence, as for $\mathrm{MnF}_{2}$ the boson field of the $\mathrm{K}_{2} \mathrm{MnF}_{4}$ sample investigated in [34] fits the $1 \mathrm{D}$ univer- sality class for half-integer spin $(S=5 / 2)$.

As a summary of our studies we present in Table I the universality classes of the GSW boson field at $T=0$ in dependence of the dimensionality of the field and of spin quantum number. The $T^{\varepsilon}$ power functions apply to thermal decrease of the magnetic order parameter and to heat capacity of the boson field. Table II gives the associated scheme of the six dispersion relations of the field quanta.

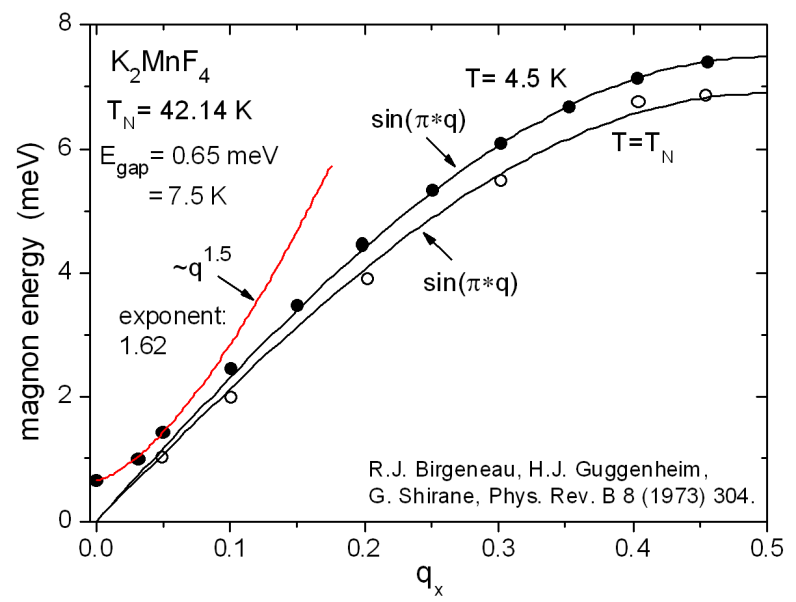

Fig. 8. Magnon dispersion in the basal plane of $\mathrm{K}_{2} \mathrm{MnF}_{4}$ at $T=4.5 \mathrm{~K}$ and at $T=T_{\mathrm{N}}=42.14 \mathrm{~K}$ [34]. Fitting power function through the first three data points of the low temperature curve results into $q^{1.62}$ dependence. This result is consistent with the $q^{1.5}$ dispersion relation attributed to the $1 \mathrm{D}$ boson field for halfinteger $\operatorname{spin}(S=5 / 2)$.

\section{Conclusions}

Observation of a significant magnon gap in magnets with isotropic spin cannot be understood on the basis of spin wave theory. For instance, in cubic magnets with integer spin large magnon gaps are found but in cubic magnets with half-integer spin virtually no magnon gaps are observed. We could identify the surprisingly strong functional anomalies in the magnon dispersions at small wave vector values as due to interactions between magnons and the boson guiding field. In particular, the magnon excitation gap seems to be essentially due to magnonGSW boson interactions. Direct magnon-GSW boson interactions request that the bosons have magnetic moment. This, in turn, requests that the GSW bosons have non-linear dispersion. In fact, in isotropic magnets with integer spin the GSW boson dispersion is $\sim q^{2}$ but in isotropic magnets with half-integer spin the dispersion of the GSW bosons is $\sim q$. This explains why a gap occurs for cubic magnets with integer spin but not for cubic magnets with half-integer spin. If the boson field has a low dimensionality, as in $\mathrm{MnF}_{2}$, its anisotropy is transferred by virtue of magnon-boson interaction to the intrinsically isotropic spin system. This leads to a spin-flop field which is much larger than can reasonably be expected for a material with pure spin magnetism. In other words, a 
large magnon gap does not always mean anisotropy but is indicative of strong magnon-GSW boson interactions only.

Since magnon-GSW boson interactions are limited to small $q$-values one has to distinguish between a boson defined magnon dispersion in the low wave vector range and an exchange defined large wave vector range. The analytical change has the character of a functional crossover. As an empirical fact, magnon dispersions in the boson defined part of the spectrum can be described by a power function of wave vector. However, the available magnon dispersion data in the boson defined part of the magnon spectrum occasionally are very limited. Precision evaluations of the exponents are not always possible. Nevertheless we could rationalize that the power function in the magnon dispersions has to be identified as the dispersion relation of the GSW bosons. This is proven by results obtained by the completely different method of resonating GSW boson states in thin ferromagnetic films [13].

As a conclusion, magnon dispersions get attracted by the boson dispersions and assume the dispersion of the boson field. Note that attractive interactions are possible between particles of different (translational) symmetry such as GSW bosons and magnons. For interactions between particles of the same (translational) symmetry such as phonons and magnons the associated dispersion lines avoid each other. Using this systematic we could evaluate the boson dispersion relations for all dimensions of the field (see Table II). The dimensionality of the field is inferred from the well established universal $T^{\varepsilon}$ functions in the thermal decrease of the order parameter, or, equivalently, from magnetic heat capacity measurements (see Table I). Since the presented results are empirical, they are without a sound theoretical justification as yet. However they can provide useful guide lines for the development of future field theories of magnetism. Final approval of the proposed power functions must come from those field theories.

\section{References}

[1] K.G. Wilson, J. Kogut, Phys. Rep. 12C, 75 (1974).

[2] K.G. Wilson, Rev. Mod. Phys. 47, 773 (1975).

[3] E. Brézin, J.C. Le Guillou, J. Zinn-Justin, Phys. Rev. B 10, 892 (1974).

[4] J.C. Le Guillou, J. Zinn-Justin, Phys. Rev. B 21, 3976 (1980).

[5] J. Goldstone, A. Salam, S. Weinberg, Phys. Rev. 127, 965 (1962).

[6] H. Ikeda, K. Hirakawa, Solid State Commun. 14, 529 (1974).

[7] E.J. Samuelsen, Phys. Rev. Lett. 31, 936 (1973).

[8] L. Onsager, Phys. Rev. 65, 117 (1944).
[9] C.N. Yang, Phys. Rev. 85, 808 (1952).

[10] U. Köbler, A. Hoser, Physica B 362, 295 (2005).

[11] U. Köbler, A. Hoser, Eur. Phys. J. B 60, 151 (2007).

[12] U. Köbler in: Recent Developments in Magnetism Research, Nova Sci. Publ., Hauppage, New York 2013, p. 1.

[13] U. Köbler, A. Hoser, Acta Phys. Pol. A 121, 1176 (2012).

[14] W.C. Koehler, H.R. Child, R.M. Nicklow, H.G. Smith, R.M. Moon, J.W. Cable, Phys. Rev. Lett. 24, 16 (1970).

[15] A. Okazaki, K.C. Turberfield, R.W.H. Stevenson, Phys. Lett. 8, 9 (1964).

[16] M.P. Schulhof, R. Nathans, P. Heller, A. Linz, Phys. Rev. B 4, 2254 (1971).

[17] A.D.B. Woods, B.N. Brockhouse, R.A. Cowley, W. Cochrane, Phys. Rev. 131, 1025 (1963).

[18] U. Köbler, A. Hoser, J. Magn. Magn. Mater. 325, 87 (2013).

[19] J.W. Stout, L.M. Matarrese, Rev. Mod. Phys. 25, 338 (1953).

[20] Y. Shapira, S. Foner, Phys. Rev. B 1, 3083 (1970).

[21] U. Köbler, A. Hoser, Renormalization Group Theory - Impact on Experimental Magnetism, Springer, Berlin 2010.

[22] R.J. Birgeneau, W.B. Yelon, E. Cohen, J. Makovsky, Phys. Rev. B 5, 2607 (1972).

[23] I.S. Jacobs, P.E. Lawrence, Phys. Rev. 164, 866 (1967).

[24] P. Martel, R.A. Cowley, R.W.H. Stevenson, Canad. J. Phys. 46, 1355 (1968).

[25] U. Köbler, A. Hoser, J.-U. Hoffmann, Physica B 382, 98 (2006).

[26] W. Jauch, M. Reehuis, A.J. Schultz, Acta Crystallogr. A 60, 51 (2004).

[27] J. Skalyo, Jr., G. Shirane, R.J. Birgeneau, H.J. Guggenheim, Phys. Rev. Lett. 23, 1394 (1969).

[28] N.D. Mermin, H. Wagner, Phys. Rev. Lett. 17, 1133 (1966).

[29] B. Schröder, V. Wagner, N. Lehner, K.M. Kesharwani, R. Geick, Phys. Status Solidi B 97, 501 (1980).

[30] M.T. Hutchings, B.D. Rainford, H.J. Guggenheim, J. Phys. C 3, 307 (1970).

[31] U. Köbler, A. Hoser, J. Magn. Magn. Mater. 349, 88 (2014).

[32] B. Hälg, A. Furrer, Phys. Rev. B 34, 6258 (1986).

[33] G.H. Lander, W.G. Stirling, Phys. Rev. B 21, 436 (1980).

[34] R.J. Bigeneau, H.J. Guggenheim, G. Shirane, Phys. Rev. B 8, 304 (1973).

[35] G.K. Wertheim, H.J. Guggenheim, H.J. Levinstein, D.N.E. Buchanan, R.C. Sherwood, Phys. Rev. 173 , 614 (1968). 